\title{
Development and characterization of self-nanoemulsifying drug delivery system (SNEDDS) formulation for enhancing dissolution of fenofibric acid
}

\author{
Wira Noviana SUHERY ${ }^{1,2}$ (D), Yeyet Cahyati SUMIRTAPURA ${ }^{1}\left(\mathbb{D}\right.$, Jessie Sofia PAMUDJI $^{1}$ (D), \\ Diky MUDHAKIR 1 * (D)
}

1 School of Pharmacy, Institut Teknologi Bandung, Ganesha 10, Bandung 40132, Indonesia.

2 Sekolah Tinggi Ilmu Farmasi Riau (STIFAR), Simpang Baru, Pekanbaru 28293, Indonesia

* Corresponding Author. E-mail: mudhakir@fa.itb.ac.id (D.M.); Tel. +62-222-504 8524.

Received: 15 May 2020 / Revised: 14 July 2020 / Accepted: 10 August 2020

\begin{abstract}
Fenofibric acid is an active form of fenofibrate and a poorly water-soluble drug. The study aims to formulate and characterize self-nanoemulsifying drug delivery system (SNEDDS) to improve the dissolution of fenofibric acid. The selection of oil, surfactant, and cosurfactant for preliminary screening was determined by the solubility study of fenofibric acid in various vehicles. The composition of selected oil, surfactant, and cosurfactant was glyceryl monolinoleate (Maisine ${ }^{\circledR}$ CC), Kolliphor ${ }^{\circledR}$ RH 40, and Transcutol ${ }^{\circledR}$ HP, PEG 400 respectively. The study results showed that the best SNEDDS formulation was Fd with a ratio of oil, surfactant, and cosurfactant of 10:70:20 \% v/v/v (Maisine ${ }^{\circledR}$ CC: Kolliphor ${ }^{\circledR}$ RH 40 : Transcutol® HP). It produced good self-nanoemulsifying drug delivery system such as the droplet size was $15.30 \mathrm{~nm}, 99.27 \%$ transmittance, thermodynamically stable, self-emulsification time was 18.84 seconds, the morphology of the formed nanoemulsion droplets was observed as sphere-shaped droplets and 1.6 fold increase in the dissolution of fenofibric acid.
\end{abstract}

KEYWORDS: Fenofibric acid; dissolution; long-chain triglyceride; Maisine ${ }^{\circledR}$ CC; SNEDDS.

\section{INTRODUCTION}

Drug solubility is the rate-limiting step of dissolution for a poorly water-soluble drug. Based on the classification of the Biopharmaceutical Classification System (BCS), drugs that belong to class II and IV have low solubility in water [1,2]. Recently, more than $40 \%$ of new drugs in the market have low solubility in water. Therefore, considerable effort has been made in increasing drug solubility leading to the improvement of its oral bioavailability $[3,4]$.

Several techniques which can be used to improve solubility and dissolution of drugs include lipid-based formulations, such as liposomes [5, 6], solid lipid nanoparticles [7, 8], nanostructured lipid carriers [9, 10], micro/nanoemulsion [11, 12], and self-nanoemulsifying drug delivery systems (SNEDDS) [13-19]. Lipid-based formulation increases the absorption of a poorly water-soluble drug from the gastrointestinal tract by 1) increasing drug solubilization (accelerate the dissolution process) 2) by recruiting intestinal lymphatic drug transport (reduced first-pass metabolism), and 3) by interacting with enterocytes-based transport processes [20].

SNEDDS consisted of an isotropic mixture of oil, surfactant, and cosurfactant, which upon dilution with aqueous phase of the gastrointestinal tract (GIT) spontaneously forms an oil/water $(\mathrm{o} / \mathrm{w})$ nanoemulsion. SNEDDS formulation has the potential to be used for delivery of lipophilic drugs (Log P $>2$ ) that are usually associated with low solubility in water, and also have a low bioavailability [21, 22]. In addition, hydrophobic drugs can be dissolved in these systems, and maintained in a dissolved state, thus, enabling them to be administrated as a unit dosage form per-oral administration $[15,18]$. SNEDDS can be presented in the form of a pre-concentrated solution. Therefore, the dissolution step required for solid crystalline compounds can be avoided. The advantages of SNEDDS compared to conventional emulsions or other lipid carriers are that they require reduced amounts of energy for their preparation and physical stability upon storage $[15,18]$.

How to cite this article: Suhery WN, Sumirtapura YC, Pamudji JS, Mudhakir D. Development and characterization of self-nanoemulsifying drug delivery system (SNEDDS) formulation for enhancing dissolution of fenofibric acid. J Res Pharm. 2020; 24(5): 738-747. 
Fenofibric acid (2-[4-(4-chloro benzoyl) phenoxy]-2-methyl propanoic acid) in Figure 1 is a BCS class II drug whose formulation can be developed using the SNEDDS formulation technology. Fenofibric acid exhibits low aqueous solubility. The poor aqueous solubility of fenofibric acid can lead to poor absorption via the oral delivery route and its bioavailability to be unpredictable [23], therefore, the required approach to improve the solubility, dissolution, and oral bioavailability of fenofibric acid with a simple, easy, and efficient technique.

Fenofibric acid is an anti-hyperlipidemia drug which is an active form of fenofibrate [23,24]. There is no use of fenofibric acid in cholesterol therapy in Indonesia because this drug has not been approved yet to be marketed in Indonesia. Currently, only a few studies on the development of fenofibric acid formulations have been reported. The methods used to improve the dissolution of fenofibric acid were solid dispersion techniques $[23,26]$.

In this study, SNEDDS containing fenofibric acid was formulated and characterized to enhance the dissolution of fenofibric acid.

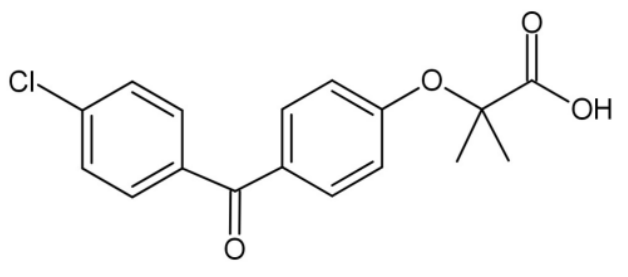

Figure 1. Chemical structure of fenofibric acid

\section{RESULTS AND DISCUSSION}

\subsection{Solubility study of fenofibric acid in oils, surfactants, and cosurfactants}

The selection of oil, surfactant, and cosurfactant was done based on the solubility of fenofibric acid in each vehicle. The solubility test done in oil showed that Maisine ${ }^{\circledR}$ CC (MCC) was the chosen oil due to the highest solubilization for fenofibric acid $(7,79 \mathrm{mg} / \mathrm{ml} \pm 0.69 \mathrm{mg} / \mathrm{ml})$. Other types of oils, namely soybean oil, virgin coconut oil, olive oil, sunflower oil, rice bran oil, oleic acid, and glyceryl monooleate have lower solubility of fenofibric acid than MCC. The result is shown in Table 1. MCC (glyceryl monolinoleate) is a winterized oil composed of long-chain $\left(\mathrm{C}_{18}\right)$ mono-, di-, and triglycerides and corn glycerides. Oil component in SNEDDS formulation play an important role in drug solubility enhancement and drug transport mechanisms [28].

The selected surfactant was Kolliphor ${ }^{\circledR}$ RH 40 (KRH 40), which can solubilize of fenofibric acid as much as $69.22 \mathrm{mg} / \mathrm{ml}$. The main constituent of KRH 40 is glycerol polyethylene glycol hydroxy-stearate, which along with fatty acid glycerol polyglycol esters, forms the hydrophobic part of the product. The hydrophilic part consists of polyethylene glycols and glycerol ethoxylate. KRH 40 has a hydrophilic-lipophilic balance (HLB) between 14 and 16 [29]. The selection of surfactants not only refers to the solubility of fenofibric acid but also depends on good emulsification capabilities. The solubility value of fenofibric acid in Tween 20, Tween 80, Kolliphor ${ }^{\circledR}$ HS 15 (KHS 15), and KRH 40 was not significantly different, in the range of $62.85-69.76 \mathrm{mg} / \mathrm{ml}$. However, KRH 40 was exhibited as the best emulsification ability among others. High HLB-value surfactants have high hydrophilicity to aid droplet formation and provide a large surface area that will promote the rapid spread of formulations in water media. Surfactants play an important role in reducing surface free energy thus, it will reduce the size of the emulsion globules formed upon SNEDDS diluted with water [14, 27, 28].

The selected cosurfactants were Transcutol ${ }^{\circledR}$ HP (THP) and polyethylene glycol 400 (PEG 400). Cosurfactants have a role in assisting fenofibric acid dissolve in the system and reduce the bending stress of the interface. They also allow an interfacial film with sufficient elasticity to assume different curvatures required to form a nanoemulsion over a wide range of compositions [28, 30].

\subsection{Construction of pseudo-ternary phase diagram}

SNEDDS formulations without containing fenofibric acid that produced nanoemulsion (globule size $<100 \mathrm{~nm}$ ) upon dilution with distilled water were constructed by a pseudo-ternary diagram. For this reason, the pseudo-ternary diagrams were constructed to identify self-nanoemulsifying regions and to select suitable concentrations of oil, surfactant, and cosurfactant for SNEDDS formulations [27, 30]. As a result, the concentration of ingredients that produced nanoemulsions for oil were of $10 \%-30 \%$, surfactants $20 \%-80 \%$, and cosurfactants were of $10 \%-70 \%$ (Figure 2). The globule size was found to be increased by increasing oil 
concentration, whereas the globule size would be decreased by increasing surfactant concentration and by decreasing cosurfactant concentration. These results were similar to the report by Kim et al., 2018 and Kanwal et al., 2019 [16, 17]. According to the pseudo-ternary diagram obtained up to eight of SNEDDS formulations containing fenofibric acid were to be characterized (Table 2). The concentration of ingredients that produced nanoemulsions after load fenofibric acid were oil $10 \%-20 \%$, surfactants $50 \%-80 \%$, and cosurfactants were of $10 \%-30 \%$.

Table 1. The solubility of fenofibric acid in oils, surfactants, and cosurfactants $(n=3)$.

\begin{tabular}{|c|c|}
\hline Component & Solubility $(\mathrm{mg} / \mathrm{ml})$ \\
\hline \multicolumn{2}{|c|}{ Oil } \\
\hline Soya oil & $0.70 \pm 0.32$ \\
\hline Virgin coconut oil & $0.95 \pm 0.27$ \\
\hline Olive oil & $0.52 \pm 0.05$ \\
\hline Sunflower oil & $0.78 \pm 0.04$ \\
\hline Rice bran oil & $1.05 \pm 0.29$ \\
\hline MCC & $7.79 \pm 0.69$ \\
\hline Oleic acid & $0.34 \pm 0.08$ \\
\hline Glyceryl monooleate & $0.29 \pm 0.13$ \\
\hline \multicolumn{2}{|c|}{ Surfactant } \\
\hline Tween 20 & $62.85 \pm 1.28$ \\
\hline Tween 80 & $66.92 \pm 1.23$ \\
\hline KRH 40 & $69.22 \pm 0.58$ \\
\hline KHS 15 & $69.76 \pm 0.20$ \\
\hline \multicolumn{2}{|c|}{ Cosurfactant } \\
\hline Propylene glycol & $20.88 \pm 0.56$ \\
\hline Glycerin & $8.76 \pm 0.52$ \\
\hline PEG 400 & $70.89 \pm 1.60$ \\
\hline THP & $88.18 \pm 0.78$ \\
\hline
\end{tabular}
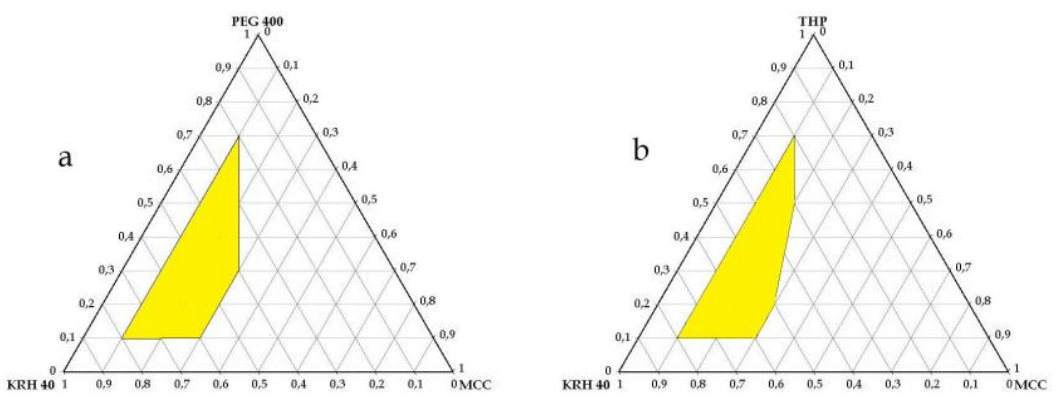

Figure 2. Pseudo-ternary diagram of SNEDDS formulations, (a) MCC-KRH 40-PEG 400, (b) MCC-KRH $40-$ THP.

\subsection{Characterization of fenofibric acid-SNEDDS}

\subsubsection{Solubility of fenofibric acid in SNEDDS formulation}

The highest solubility of fenofibric acid in the SNEDDS formulation was $60.92 \pm 2.15 \mathrm{mg} / \mathrm{ml}$ (Fd). While the smallest amount of fenofibric acid that can dissolve in SNEDDS was $42.20 \pm 5.56 \mathrm{mg} / \mathrm{ml}(\mathrm{Fc})$. These results indicated that the solubility of fenofibric acid in SNEDDS was influenced by the ratio of oil, surfactants, and cosurfactants in SNEDDS. The use of THP as a cosurfactant showed better improvement of solubility of fenofibric acid in SNEDDS formulation compared to that of PEG 400. All of the solubility data is shown in Table 3. The high solubility of a drug in SNEDDS formulation reduces the potential for drug precipitation while SNEDDS is diluted with water [27]. 
Table 2. Formulations of fenofibric acid-SNEDDS

\begin{tabular}{cccc}
\hline \multirow{2}{*}{ Formulation } & Oil (\%) & Surfactant (\%) & Cosurfactant (\%) \\
\cline { 2 - 4 } & MCC & KRH 40 & PEG 400 \\
\hline Fa & 10 & 70 & 20 \\
Fb & 10 & 80 & 10 \\
Fc & 20 & 70 & 10 \\
\hline & MCC & KRH 40 & THP \\
\hline Fd & 10 & 70 & 20 \\
Fe & 10 & 80 & 10 \\
Ff & 20 & 50 & 30 \\
Fg & 20 & 60 & 20 \\
Fh & 20 & 70 & 10 \\
\hline
\end{tabular}

Table 3. Characterization of fenofibric acid-SNEDDS ( $n=3)$

\begin{tabular}{cccccc}
\hline Formulation & $\begin{array}{c}\text { Solubility } \\
(\mathbf{m g} / \mathbf{m l})\end{array}$ & $\begin{array}{c}\text { Globule size } \\
\mathbf{( n m})\end{array}$ & $\begin{array}{c}\text { Polydispersity } \\
\text { index }\end{array}$ & $\begin{array}{c}\text { Zeta potential } \\
\mathbf{( m V})\end{array}$ & $\begin{array}{c}\text { Percentage of } \\
\text { transmittance } \\
(\%)\end{array}$ \\
\hline $\mathrm{Fa}$ & $42.54 \pm 5.93$ & $17.90 \pm 1.90$ & $0.29 \pm 0.32$ & $-20.57 \pm 0.75$ & $99.17 \pm 0.15$ \\
$\mathrm{Fb}$ & $42.70 \pm 0.86$ & $14.07 \pm 2.50$ & $0.09 \pm 0.08$ & $-18.70 \pm 0.45$ & $99.30 \pm 0.10$ \\
$\mathrm{FC}$ & $42.20 \pm 5.56$ & $41.13 \pm 1.05$ & $0.35 \pm 0.01$ & $-19.77 \pm 0.77$ & $95.10 \pm 0.10$ \\
$\mathrm{Fd}$ & $60.92 \pm 2.15$ & $15.30 \pm 4.32$ & $0.09 \pm 0.09$ & $-17.50 \pm 1.55$ & $99.27 \pm 0.05$ \\
$\mathrm{Fe}$ & $52.59 \pm 4.61$ & $14.67 \pm 1.28$ & $0.11 \pm 0.05$ & $-19.40 \pm 1.05$ & $99.57 \pm 0.05$ \\
$\mathrm{Ff}$ & $59.34 \pm 5.61$ & $50.70 \pm 1.15$ & $0.39 \pm 0.01$ & $-19.47 \pm 0.81$ & $89.85 \pm 0.10$ \\
$\mathrm{Fg}$ & $46.96 \pm 0.62$ & $41.13 \pm 1.75$ & $0.36 \pm 0.04$ & $-17.13 \pm 0.05$ & $95.10 \pm 0.26$ \\
$\mathrm{Fh}$ & $45.06 \pm 5.86$ & $40.23 \pm 7.11$ & $0.37 \pm 0.10$ & $-20.87 \pm 0.95$ & $95.57 \pm 0.15$ \\
\hline
\end{tabular}

\subsubsection{Globule size, polydispersity index, and zeta potential}

The globule size and the polydispersity index of all fenofibric acid-SNEDDS (Fa-Fh) formulations, after being diluted 100 times [14, 18, 31] with distilled water, were $<100 \mathrm{~nm}$ which ranged from $14.07 \pm 2.50 \mathrm{~nm}$ to $50.70 \pm 1.15 \mathrm{~nm}$; whereas, the values of the polydispersity index of all formulations were $<0.4$, which ranges from $0.09 \pm 0.09$ to $0.39 \pm 0.01$. The polydispersity index varies from $0.0-1.0$. The closer to zero the polydispersity index value, the more the particles are homogenous [30]. These results indicate that fenofibric acid-SNEDDS formulations have a homogenous droplet population and uniform nanoemulsions. Determination of the zeta potential value aimed to see the distance between the globule particles and to indicate the potential stability of the colloidal system [30]. The farther the distance between globule particle charges, the more stable an emulsion will be because it prevents coalescence. The critical zeta potential values for stable emulsion were more positive than $+30 \mathrm{mV}$ or more negative than $-30 \mathrm{mV}$. All formulations of fenofibric acid-SNEDDS (Fa-Fh) obtained from this study have zeta potential values ranging from $-17.13 \mathrm{mV}$ to $-20.87 \mathrm{mV}$. It was indicated that the value of zeta potential was lower than the values reported in the literature. This might be attributed to the presence of KRH 40 and THP which sterically stabilize the system by forming a coat around their surface [30]. These results are in alignment with the results reported by Nasr et al., 2016 and Kaseem et al., 2016. The negative zeta potential values were due to the presence of fatty acid and nonionic surfactant in SNEDDS formulations [15]. The zeta potential value of all formulations can be seen in Table 3.

\subsubsection{Percentage of transmittance}

The percent transmittance value indicates the level of clarity of an emulsion. If the transmittance value of an emulsion is closer to $100 \%$, the emulsion reflects in the range of nano-size globules. All formulations (FaFh) showed percentages of transmittance ranging from approximately $89-99 \%$. This result indicated that the globule size of all formulation at size $<100 \mathrm{~nm}$ (Table 3). 


\subsubsection{Self-emulsification time}

The time of self-emulsification showed the time required of SNEDDS formulation to form a homogenous mixture upon dilution under mild agitation. It has been reported that the mechanism of selfemulsification involves the erosion of a fine cloud of small droplets from the surface of large droplets, rather than the progressive reduction in droplet size [31,32]. The emulsification time of fenofibric acid-SNEDDS ranged from $18.84 \pm 1.06$ to $142.82 \pm 2.43$ seconds (Table 4). These results showed that the higher the amount of oil, the longer the emulsification time; conversely, the higher the amount of cosurfactant, the faster the emulsification time. Basalious et al., 2010 reported a similar result, in which rapid emulsification is correlated with lower content of oil and higher content of cosurfactant [33]. The reason is the increase in interfacial tension between the larger volume of oil and aqueous phase with a net decrease in surfactant system, which the emulsification times longer [31]. The formula of $\mathrm{Fd}$ was given the fastest emulsification time compared to other formulations.

Table 4. Characterization of fenofibric acid-SNEDDS after dilution 100 fold $(n=3)$.

\begin{tabular}{cccccccc}
\hline Formulation & $\begin{array}{c}\text { Self } \\
\text { emulsification } \\
\text { time (second) }\end{array}$ & $\begin{array}{c}\text { Rox } \\
\end{array}$ & $\mathbf{1 0 0 x}$ & $\mathbf{2 5 0 x}$ & Heating-cooling & Centrifugation \\
\hline $\mathrm{Fa}$ & $44.17 \pm 1.27$ & $\sqrt{ }$ & $\sqrt{ }$ & $\sqrt{ }$ & $\sqrt{ }$ & $\sqrt{ }$ \\
$\mathrm{Fb}$ & $44.84 \pm 1.38$ & $\sqrt{ }$ & $\sqrt{ }$ & $\sqrt{ }$ & $\sqrt{ }$ & $\sqrt{ }$ \\
$\mathrm{FC}$ & $142.82 \pm 2.43$ & $\sqrt{ }$ & $\sqrt{ }$ & $\sqrt{ }$ & $\sqrt{ }$ & $\sqrt{ }$ \\
$\mathrm{Fd}$ & $18.84 \pm 1.06$ & $\sqrt{ }$ & $\sqrt{ }$ & $\sqrt{ }$ & $\sqrt{ }$ & $\sqrt{ }$ \\
$\mathrm{Fe}$ & $38.05 \pm 1.71$ & $\sqrt{ }$ & $\sqrt{ }$ & $\sqrt{ }$ & $\sqrt{ }$ & $\sqrt{ }$ \\
$\mathrm{Ff}$ & $23.82 \pm 5.61$ & $\sqrt{ }$ & $\sqrt{ }$ & $\sqrt{ }$ & $\sqrt{ }$ & $\sqrt{ }$ \\
$\mathrm{Fg}$ & $79.24 \pm 4.30$ & $\sqrt{ }$ & $\sqrt{ }$ & $\sqrt{ }$ & $\sqrt{ }$ & $\sqrt{ }$ \\
$\mathrm{Fh}$ & $119.47 \pm 5.79$ & $\sqrt{ }$ & $\sqrt{ }$ & $\sqrt{ }$ & $\sqrt{ }$ & \multicolumn{2}{c}{ Physical stability accelerated } \\
\hline
\end{tabular}

$\checkmark$ : Stable and globule size in nano-range $(<100 \mathrm{~nm})$

\subsubsection{Robustness to dilution}

Robustness to dilution aims to show whether SNEDDS will continue to form stable nanoemulsions after being diluted with distilled water. From the observations, it was found that all formulations were still in the form of nanoemulsions ( $<100 \mathrm{~nm}$ ), and no sign of phase separation showed, after 50, 100, and 250 times dilutions respectively. The result is shown in Table 4.

\subsubsection{Accelerated physical stability test}

Accelerated stability testing included heating-cooling and centrifuge methods were used to assess the stability of SNEDDS. The test was carried out to observe whether SNEDDS was still stable after being stored in extreme conditions. The result showed that all formulations were stable marked by the absence of separation and the size of the globule particles after being diluted with distilled water was still in the nano range $(<100 \mathrm{~nm})$. The result can be seen in Table 4 .

\subsubsection{In vitro dissolution test}

In vitro dissolution test of fenofibric acid-SNEDDS was carried out to determine the dissolution of fenofibric acid from SNEDDS formulations. All SNEDDS formulations revealed a higher dissolution percentage compared to the pure fenofibric acid. Within the initial 30 minutes, the formulation of $\mathrm{Fa}, \mathrm{Fb}, \mathrm{Fd}$, $\mathrm{Fe}, \mathrm{Ff}, \mathrm{Fg}$ can dissolve and release up to $86.01 \%$ to $97.77 \%$ of fenofibric acid whereas the pure drug can only release $56.26 \%$ of fenofibric acid. After 60 minutes, all formulations dissolved and released about approximately $99 \%$, while the dissolution percentage of the pure drug was only $59.03 \%$. This means that the preparation of fenofibric acid in the form of SNEDDS can improve its solubility significantly. The percentage of dissolution of each formulation are shown in Figure 3. The higher the surfactant concentration in the formulation, the more the increase in percent dissolution of fenofibric acid. The higher the concentration of oil, the slower the dissolution of fenofibric acid. Besides, the dissolution also was related to the emulsification time in each formulation. Formulations that have a faster emulsification time lead to the higher dissolution of fenofibric acid especially within the initial ten minutes. In line to this, the emulsification time of Fc and Fh 
were longer than other formulations, which may be attributed to the lower percentage of dissolution. Fenofibric acid in SNEDDS formulations was in the dissolved state when contacted with the dissolution media. The fenofibric acid was immediately transferred to the dissolution media, thus, it enhanced drug dissolution rate and extent $[18,30]$. Similar observations were reported by Senapati et al., 2016, Syukri et al., 2018, and Nasr et al., 2016, that showed that the release of the drug (efavirenz, andrographolide, and olmesartan medoxomil) from selected SNEDDS was found to be significantly higher as compared with the pure drug [14, 19, 30]. Based on the evaluation of all characteristics, it was found that the best formulation was Fd.

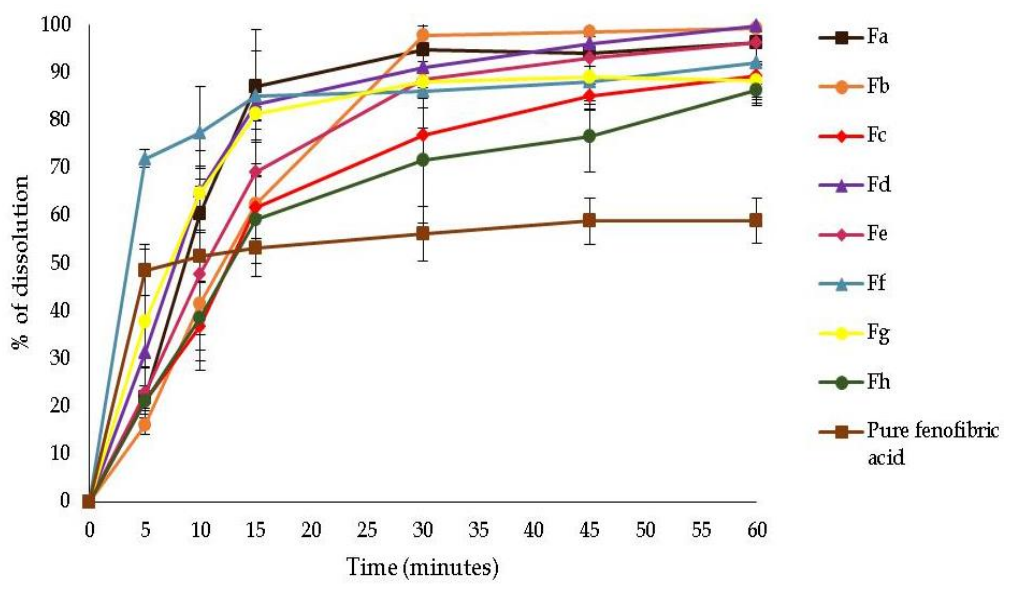

Figure 3. Profil of dissolution of fenofibric acid-SNEDDS formulations $(n=3)$.

\subsubsection{Surface morphology determination of fenofibric acid-SNEDDS}

Investigation of surface morphology of fenofibric acid-SNEDDS globules after being diluted with distilled water was conducted by using Transmission Electron Microscope (TEM, Jeol 1010, Tokyo, Japan). The shapes of the globules were spherical with a diameter of $<100 \mathrm{~nm}$. The size of globules was uniform and there was a clear boundary that was seen around globule particles. The existence of a boundary line around globule particles was a barrier that prevented coalescence (Figure 4). These results were similar to the report by Salem et al., 2018 [27].

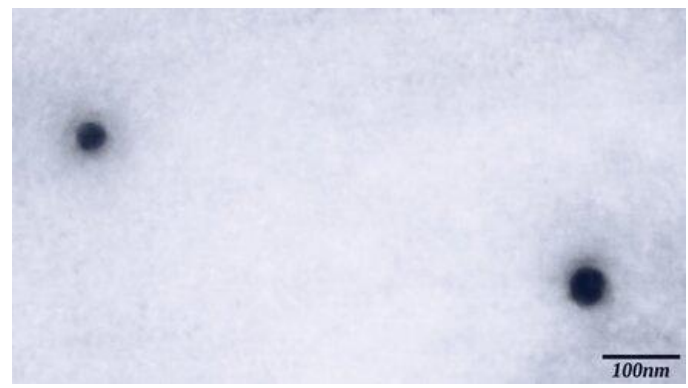

Figure 4. TEM photograph of fenofibric acid-SNEDDS formulation (Fd), magnification 50,000 x.

\subsubsection{Fourier-transform infrared spectroscopy (FT-IR).}

FT-IR analysis was carried out to identify possible interactions of SNEDDS components with fenofibric acid. The fenofibric acid spectrum showed the characteristics of wide peaks at wavenumber $2,991 \mathrm{~cm}^{-1}(\mathrm{O}-\mathrm{H}$ strain), indicating the presence of carboxylic acid groups in the structure of fenofibric acid. The peaks at 1,701 $\mathrm{cm}^{-1}$ (strain $\mathrm{C}=\mathrm{O}$ ) and 1,645 $\mathrm{cm}^{-1}$ (symmetrical strain $\mathrm{C}-\mathrm{C}=\mathrm{C}$ ) showed the functional groups of alkenes and ketones. The peaks at 1,595 $\mathrm{cm}^{-1}$ and $1,500 \mathrm{~cm}^{-1}$ (symmetrical strain $\mathrm{C}-\mathrm{C}=\mathrm{C}$ ) showed aromatic rings, and the vibrations at $758 \mathrm{~cm}^{-1}$ and $671 \mathrm{~cm}^{-1}$ showed alkyl halide $(\mathrm{C}-\mathrm{Cl})$ groups. The small peaks of fenofibric acid appeared in the Fd spectrum with wavenumbers at around $1,645 \mathrm{~cm}^{-1}$ showed $C=C$ aromatic strains $(1,675-$ $\left.1,500 \mathrm{~cm}^{-1}\right)$, and strains $\mathrm{C}-\mathrm{Cl}$ at wavenumbers around $671 \mathrm{~cm}^{-1}$. Furthermore, the appearance of an extra peak in the spectrum of Fd at $2857 \mathrm{~cm}^{-1}$ and $2924 \mathrm{~cm}^{-1}$ of $-\mathrm{CH}$ stretching showed the contribution of surfactant [14]. FT-IR analysis showed that fenofibric acid did not interact chemically with components in SNEDDS formulation (Figure 5). 


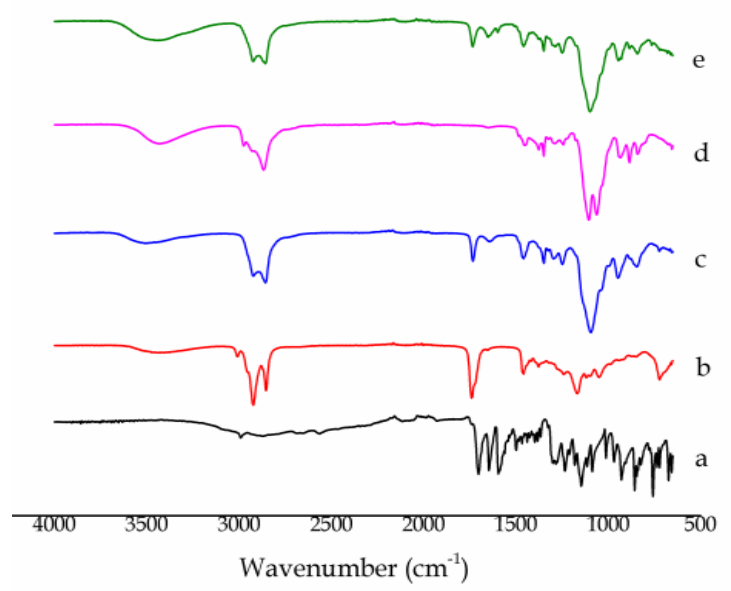

Figure 5. FT-IR spectra of (a) fenofibric acid, (b) MCC, (c) KRH 40, (d) THP, (e) fenofibric acid-SNEDDS (Fd).

\section{CONCLUSION}

In this study, fenofibric acid was successfully formulated and characterized in SNEDDS formulation to increase the dissolution of fenofibric acid. SNEDDS formulations were constructed based on the pseudoternary diagram, physical properties, and the dissolution test. From this study, it was concluded that the selection of the right type and concentration of oil, surfactant, and cosurfactant can determine the success of SNEDDS formulations, characterization, and dissolution of fenofibric acid. The increase in the dissolution of fenofibric acid in SNEDDS formulations is influenced by the globule size, self-emulsification time, stability, and the solubility of the drug in the system. The drug once dissolved in the SNEDDS formulation will be emulsified in nano-size droplets quickly when in contact with GIT liquid. Thus, it will provide a large interfacial surface area for drug release, which in turn can affect the process of absorption of fenofibric acid into the blood. Finally, SNEDDS formulation represented an interesting approach to enhancing the dissolution of fenofibric acid.

\section{MATERIALS AND METHODS}

\subsection{Materials}

Fenofibric acid was purchased from BOC Sciences (New York, USA), MCC (glyceryl monolinoleate) and THP (diethylene glycol monoethyl ether) were kindly gifted from Gattefossé (Lyon, France), KRH 40 (PEG-40 hydrogenated castor oil), KHS 15 (PEG [15] hydroxy stearate) and glyceryl monooleate were kindly gifted from Badische Anilin und Soda Fabrik (BASF) (Ludwigshafen, Germany). Tween 20 (polysorbate 20), Tween 80 (polysorbate 80), propylene glycol, and glycerin were purchased from PT. Brataco Chemica (Bandung, Indonesia). Virgin coconut oil, soya oil, sunflower oil, olive oil, and oleic acid were purchased from Bandung, Indonesia. Polyethylene glycol 400 (PEG 400), potassium dihydrogen phosphate $\left(\mathrm{KH}_{2} \mathrm{PO} 4\right)$, sodium hydroxide ( $\mathrm{NaOH})$, and methanol were purchased from Merck (Darmstadt, Germany). All solvents used were analytical grade.

\subsection{Solubility study of fenofibric acid in oils, surfactants, and cosurfactants}

The solubility of fenofibric acid was determined in various oils, i.e., soya oil, virgin coconut oil, olive oil, sunflower oil, rice bran oil, MCC, oleic acid, and glyceryl monooleate. An excess of fenofibric acid was added to $1 \mathrm{ml}$ of each vehicle in glass tubes. The suspension was mixed using a vortex mixer for $2 \mathrm{~min}$ and equilibrated at $25^{\circ} \mathrm{C}$ in a water bath shaker at $100 \mathrm{rpm}$ for $72 \mathrm{~h}$. After equilibrium, the tubes were centrifuged at 5,000 rpm for $20 \mathrm{~min}$. The supernatants were solubilized with methanol and then the amount of fenofibric acid dissolved in each vehicle was analyzed via ultraviolet (UV) spectrophotometer validated method (UVVis, Beckman DU 720) at a maximum wavelength of $300 \mathrm{~nm}$. The validated UV spectrophotometer showed that $\mathrm{r}=0.999$ (with $5-17.5 \mu \mathrm{g} / \mathrm{ml}$ of calibration curve concentrations range), precision $=1.61 \%$ and $0.15 \%$, accuracy $=96.90 \%$ and $100.87 \%$. Determination of solubility of fenofibric acid in surfactants (Tween 20, Tween 80, KHS 15, KRH 40), and cosurfactants (propylene glycol, glycerin, PEG 400, THP) were conducted in the same procedure as described above [14]. 


\subsection{Construction of pseudo-ternary phase diagram}

The selected of oil, surfactant, and cosurfactant were MCC, KRH 40, and THP, PEG 400, respectively. All ingredients were mixed in the ratio of oil: Smix (surfactant and cosurfactant) was 1:9, 2:8, 3:7, 4:6, 5:5, 6:4, 7:3, 8:2, and 9:1. The composition of SNEDDS with ratios of oil: Smix that produced nanoemulsion (globule size $<100 \mathrm{~nm}$ ) was plotted on a pseudo-ternary diagram using ProSim software (Labege, France) [27].

\subsection{Preparation of fenofibric acid-SNEDDS}

The oil, surfactant, and cosurfactant were mixed into a vial and stirred by using vortex-mixer at 3,000 $\mathrm{rpm}$ for $1 \mathrm{~min}$. Then, $35 \mathrm{mg}$ of fenofibric acid was added to the mixture, and stirring is continued for $2 \mathrm{~min}$. Thereafter, the mixture was stirred using a magnetic stirrer at $500 \mathrm{rpm}$ at $40^{\circ} \mathrm{C}$ for $30 \mathrm{~min}$ until a clear solution was obtained.

\subsection{Characterization of fenofibric acid-SNEDDS}

\subsubsection{Study of fenofibric acid solubility in SNEDDS formulations}

About $200 \mathrm{mg}$ of fenofibric acid is added to $1 \mathrm{ml}$ of each SNEDDS formulation (Table 2). The drug suspension was mixed using a vortex mixer for $2 \mathrm{~min}$ and equilibrated at $25^{\circ} \mathrm{C}$ in a water bath shaker at 100 $\mathrm{rpm}$ for $72 \mathrm{~h}$. After equilibrium is achieved, the tubes are centrifuged at 5,000 rpm for $20 \mathrm{~min}$. The supernatants were solubilized with methanol and analyzed for fenofibric acid with a spectrophotometer (UV-Vis, Beckman DU 720) at a maximum wavelength of $300 \mathrm{~nm}$.

\subsubsection{Globule size analyze, polydispersity index and zeta potential}

About $0.1 \mathrm{ml}$ of SNEDDS was diluted in $10 \mathrm{ml}$ of distilled water. The droplet size and polydispersity index were measured using a Delsa ${ }^{\mathrm{TM}}$ Nano Particle Analyzer (Beckman Coulter, USA), while zeta potential measurements were performed using a Malvern ${ }^{\circledR}$ Zetasizer Nano ZSP (England).

\subsubsection{Percentage of transmittance}

About $0.1 \mathrm{ml}$ of SNEDDS was diluted with $10 \mathrm{ml}$ of distilled water, then the clarity of the nanoemulsion was measured as the percentage of transmittance at $\lambda \max$ of $500 \mathrm{~nm}$, using distilled water as the blank sample on a UV-Vis spectrophotometer (Beckman DU 720) [14].

\subsubsection{Self-emulsification time}

Self-emulsification time was determined by diluting of $0.1 \mathrm{ml}$ of fenofibric acid-SNEDDS with $10 \mathrm{ml}$ distilled water at $37^{\circ} \mathrm{C}$ and mixing gently using a magnetic stirrer with a rotation speed of $100 \mathrm{rpm}$. The time required to self-emulsify completely to form a homogenous nanoemulsion was recorded by a stopwatch.

\subsubsection{Robustness for dilution}

As much as $0.1 \mathrm{ml}$ of SNEDDS was diluted with distilled water for 50, 100, 250, and 1000 times. The diluted systems were mixed using a magnetic stirrer to complete homogeneity. The droplet size and any signs of phase separation were also observed visually.

\subsubsection{Accelerated physical stability test}

The heating-cooling cycle method was employed to study the stability of SNEDDS. Six heating-cooling cycles were carried out at $4{ }^{\circ} \mathrm{C}$ and $40^{\circ} \mathrm{C}$ for $48 \mathrm{~h}$. All formulations were observed for separation, particle size globules, creaming, and coalescence after dilution with distilled water [14]. Another stability test was done by using the centrifugation method. All formulations were centrifuged at a speed of 5,000 rpm for 30 minutes and observed for separation [14].

\subsubsection{In vitro dissolution test}

In vitro dissolution testing of fenofibric acid-SNEDDS was performed using USP dissolution apparatus I (Hanson Virtual Instruments SR8 Plus) in $900 \mathrm{ml}$ of simulated intestinal fluid media (SIFsp, pH 6.8 without enzyme) at a rotation speed of $100 \mathrm{rpm}$ temperature of $37 \pm 0.5^{\circ} \mathrm{C}[19,23]$. Formulation of Fa-Fh was placed in hard gelatin capsules $[13,14,19,30]$ prior to beginning of the dissolution test. Then $5 \mathrm{ml}$ of samples were taken at interval times of 5, 10, 15, 30, 45, and $60 \mathrm{~min}$. Immediately after each sampling, $5 \mathrm{ml}$ of new dissolution media was added to replace the volume of the sample drawn. The amount of fenofibric acid dissolved in the medium was analyzed by using a UV-Vis spectrophotometer at a maximum wavelength of $298 \mathrm{~nm}$. 


\subsubsection{Surface morphology determination of fenofibric acid-SNEDDS}

The fenofibric acid-SNEDDS were diluted 100 times with distilled water to form a nanoemulsion. A drop of nanoemulsion was applied to a copper grid and left for $10 \mathrm{~min}$ to allow the particles to bind to collodion and the excess was removed. A drop of $2 \%$ of the uranyl acetate solution was then applied. The excess was removed and the samples were air-dried for $1 \mathrm{~min}$, then observed under TEM (Jeol 1010, Tokyo, Japan).

\subsubsection{Fourier-transform infrared spectroscopy (FT-IR)}

The Fourier-transform infrared (FT-IR) spectrum of each sample was performed using IR Agilent Cary 630 (USA) and analyzed within a wavelength of $500-4,000 \mathrm{~cm}^{-1}$ with a resolution of $2 \mathrm{~cm}^{-1}$. Afterwards, the peak of the obtained spectrum was compared. The intensity and shift of the vibration peak were observed.

Acknowledgements: This work was supported by the Institute for Research and Community Services of Institut Teknologi Bandung (ITB), Indonesia under Grant of Research, Community Services, and Innovation (P3MI) Program (no. 902b/SK/11.C03/PL/2018).

Author contributions: Concept - W.N.S., D.M., J.S.P., Y.C.S.; Design - W.N.S., D.M., J.S.P., Y.C.S.; Supervision - Y.C.S., J.S.P., D.M.; Resources - W.N.S., D.M.; Materials - W.N.S., J.S.P.; Data Collection and/or Processing - W.N.S.; Analysis and/or Interpretation - W.N.S., D.M., J.S.P., Y.C.S.; Literature Search - W.N.S., D.M.; Writing - W.N.S., D.M., J.S.P., Y.C.S.; Critical Reviews - W.N.S., D.M., J.S.P., Y.C.S.

Conflict of interest statement: The authors declare no conflict of interest, financial or otherwise.

\section{REFERENCES}

[1] Wu CY, Benet LZ. Predicting drug disposition via application of BCS: transport/absorption/elimination interplay and development of a biopharmaceutics drug disposition classification system. Pharm Res. 2005; 22(1): 11-23. [CrossRef]

[2] Takano R, Furumoto K, Shiraki K, Takata N, Hayashi Y, Aso Y, Yamashita S. Rate-limiting steps of oral absorption for poorly water-soluble drugs in dogs; prediction from a miniscale dissolution test and a physiologically-based computer simulation. Pharm Res. 2008; 25(10): 2334-2344. [CrossRef]

[3] Bohr A, Kristensen J, Stride E, Dyas M, Edirisinghe M. Preparation of microspheres containing low solubility drug compound by electrohydrodynamic spraying. Int J Pharm. 2011; 412(1-2): 59-67. [CrossRef]

[4] Lipinski CALF. Poor aqueous solubility - an industry wide problem in drug discovery. Am Pharm Rev. 2002; 5(3): $82-85$.

[5] Ong SGM, Ming LC, Lee KS, Yuen KH. Influence of the encapsulation efficiency and size of liposome on the oral bioavailability of griseofulvin-loaded liposomes. Pharmaceutics. 2016; 8(3): 25. [CrossRef]

[6] Pauli G, Tang WL, Li SD. Development and characterization of the solvent-assisted active loading technology (SALT) for liposomal loading of poorly water-soluble compounds. Pharmaceutics. 2019; 11(9): 465. [CrossRef]

[7] Yasmin R, Rao S, Bremmell KE, Prestidge CA. Porous silica-supported solid lipid particles for enhanced solubilization of poorly soluble drugs. The AAPS J. 2016: 18(4): 876-885. [CrossRef]

[8] Kumar R, Singh A, Sharma K, Dhasmana D, Garg N, Siril PF. Preparation, characterization and in vitro cytotoxicity of fenofibrate and nabumetone loaded solid lipid nanoparticles. Mater Sci Eng C. 2020; 106: 110-184. [CrossRef]

[9] Mishra A, Imam SS, Aqil M, Ahad A, Sultana Y, Ameeduzzafar, Ali A. Carvedilol nano lipid carriers: formulation, characterization and in-vivo evaluation. Drug Deliv. 2016; 23(4): 1486-1494. [CrossRef]

[10] Choi KO, Choe J, Suh S, Ko S. Positively charged nanostructured lipid carriers and their effect on the dissolution of poorly soluble drugs. Molecules. 2016; 21(5): 672. [CrossRef]

[11] Li H, Pan T, Cui Y, Li X, Gao J, Yang W, Shen S. Improved oral bioavailability of poorly water-soluble glimepiride by utilizing microemulsion technique. Int J Nanomedicine. 2016; 11: 3777. [CrossRef]

[12] Wik J, Bansal KK, Assmuth T, Rosling A, Rosenholm JM. Facile methodology of nanoemulsion preparation using oily polymer for the delivery of poorly soluble drugs. Drug Deliv Transl Res. 2019: 1-13. [CrossRef]

[13] Kamble RN, Mehta PP, Kumar A. Efavirenz self-nano-emulsifying drug delivery system: in vitro and in vivo evaluation. AAPS Pharm Sci Tech. 2016; 17(5): 1240-1247. [CrossRef] 
[14] Senapati PC, Sahoo SK, Sahu AN. Mixed surfactant based (SNEDDS) self-nanoemulsifying drug delivery system presenting efavirenz for enhancement of oral bioavailability. Biomed Pharmacother. 2016; 80: 42-51. [CrossRef]

[15] Kassem AA, Mohsen AM, Ahmed RS, Essam TM. Self-nanoemulsifying drug delivery system (SNEDDS) with enhanced solubilization of nystatin for treatment of oral candidiasis: Design, optimization, in vitro and in vivo evaluation. J Mol Liq. 2016; 218: 219-232. [CrossRef]

[16] Kim RM, Jang DJ, Kim YC, Yoon JH, Min KA, Maeng HJ, Cho KH. Flurbiprofen-loaded solid SNEDDS preconcentrate for the enhanced solubility, in-vitro dissolution and bioavailability in rats. Pharmaceutics. 2018; 10(4): 247. [CrossRef]

[17] Kanwal T, Kawish M, Maharjan R, Shah MR, et al. Design and development of permeation enhancer containing selfnanoemulsifying drug delivery system (SNEDDS) for ceftriaxone sodium improved oral pharmacokinetics. J Mol Liq. 2019; 289: 111098. [CrossRef]

[18] Balakumar K, Raghavan CV, Abdu S. Self-nanoemulsifying drug delivery system (SNEDDS) of rosuvastatin calcium: design, formulation, bioavailability, and pharmacokinetic evaluation. Colloids Surf B. 2013; 112: 337-343. [CrossRef]

[19] Syukri Y, Martien R, Lukitaningsih E, Nugroho AE. Novel self-nanoemulsifying drug delivery system (SNEDDS) of andrographolide isolated from Andrographis paniculata Ness: characterization, in-vitro and in-vivo assessment. J Drug Deliv Sci Tech. 2018; 47: 514-520. [CrossRef]

[20] Porter C, Trevaskis N, Charman W. Lipids and lipid-based formulations: optimizing the oral delivery of lipophilic drugs. Nat Rev Drug Discov. 2007; 6: 231-248. [CrossRef]

[21] Pouton CW. Formulation of poorly water-soluble drugs for oral administration: physicochemical and physiological issues and the lipid formulation classification system. Eur J Pharm Sci. 2006; 29(3-4): 278-287. [CrossRef]

[22] Weerapol Y, Limmatvapirat S, Sriamornsak P. Effect of Lipophilicity of Drugs on Dissolution Profiles of Selfnanoemulsifying Drug Delivery System. In Adv Mat Res. Trans Tech Publications Ltd. 2015; 1060: 37-40. [CrossRef]

[23] Windriyati YN, Sumirtapura YC, Pamudji JS. Dissolution enhancement and physicochemical characterization of fenofibric acid in surface solid dispersion with croscarmellose sodium. J Res Pharm. 2019; 23(2): 315-325. [CrossRef]

[24] Moutzouri E, Kei A, Elisaf MS, Milionis HJ. Management of dyslipidemias with fibrates, alone and in combination with statins: role of delayed-release fenofibric acid. Vasc Health Risk Manag. 2010; 6: 525-539. [CrossRef]

[25] Rath NP, Haq W, Balendiran GK. Fenofibric acid. Acta Cryst C: Crystal Structure Communications. 2005; 61(2): o81-o84.

[26] Yousaf AM, Ramzan M, Shahzad Y, Mahmood T, Jamshaid M. Fabrication and in vitro characterization of fenofibric acid-loaded hyaluronic acid-polyethylene glycol polymeric composites with enhanced drug solubility and dissolution rate. Int J Polym Mater. 2019; 68(9): 510-5. [CrossRef]

[27] Salem HF, Kharshoum RM, Halawa AKA, Naguib DM. Preparation and optimization of tablets containing a selfnanoemulsifying drug delivery system loaded with rosuvastatin. J Liposome Res. 2018; 28(2):149-160. [CrossRef]

[28] Chatterjee B, Hamed AS, Ahmed MAA, Mandal UK, Sengupta P. Controversies with self-emulsifying drug delivery system from pharmacokinetic point of view. Drug Deliv. 2016; 23(9): 3639-3652. [CrossRef]

[29] BASF. Technical information Kolliphor $®$ RH 40. https://products.basf.com/documents/pim;save/en/8952336787925.Technical\%20Information.pdf (accessed on 2 March 2020).

[30] Nasr A, Gardouh A, Ghorab M. Novel solid self-nanoemulsifying drug delivery system (S-SNEDDS) for oral delivery of olmesartan medoxomil: design, formulation, pharmacokinetic and bioavailability evaluation. Pharmaceutics. 2016; 8(3), 20. [CrossRef]

[31] Agrawal AG, Kumar A, Gide PS. Formulation of solid self- nanoemulsifying drug delivery systems using N-methyl pyrrolidone as cosolvent. Drug Dev Ind Pharm. 2014; 41(4): 594-604.[CrossRef]

[32] Singh SK, Verma PRP, Razdan B. Development and characterization of a carvedilol-loaded self-microemulsifying delivery system. Clin Res Regul Aff. 2009; 26(3): 50-64. [CrossRef]

[33] Basalious EB, Shawky N, Badr-eldin SM. SNEDDS containing bioenhancers for improvement of dissolution and oral absorption of lacidipine. I: development and optimization. Int J Pharm. 2010; 391(1-2): 203-211. [CrossRef] 